\title{
EL BIENESTAR SOCIAL Y EL BUEN VIVIR: LOS OLVIDADOS EN LA TOMA DE DECISIONES EN MATERIA ECONÓMICA*
}

\author{
SOCIAL WELFARE AND THE "BUEN VIVIR": \\ THE FORGOTTEN IN ECONOMIC DECISION-MAKING
}

\author{
Geneviève Koubi \\ Université Paris-Vincennes-Saint Denis, Francia
}

\section{Resumen}

El «buen vivir» designa un paradigma inherente al campo social que busca centrarse en valores humanos como la solidaridad y el modelo de desarrollo sustentable; en este sentido, se diferencia del «vivir mejor», que es una interpretación individualista de la vida, una vida alejada del respeto por el entorno natural y que diviniza la actividad económica. Por ello, esta contribución busca introducir un marco conceptual sobre el «buen vivir», para entender que la función operativa del bienestar social no puede abordarse unidimensionalmente considerando solo la economía, sino también variables medioambientales, como la salud pública, la calidad de vida, la integración social, el acceso a los derechos sociales, etc.; lo cual impacta en la identificación de indicadores que midan también estas variables, con el objetivo de permitir sosteniblemente que todas las personas puedan ser actores del desarrollo social, político y económico.

Palabras clave: buen vivir, bienestar social, vivir bien, economicismo del derecho, derecho a medioambiente sano, desarrollo sostenible, ecosocialismo.

* Traducción del original en francés: Doctora Beatriz Collantes Sánchez (Universidad de ParísVincennes-Saint Denis, Francia). 


\begin{abstract}
The "buen vivir" designates a paradigm inherent to the social field which seeks to focus on human values such as solidarity and the model of sustainable development. In this sense, it differs from "living better" — an individualistic interpretation of life- - far from respecting the natural environment and which deifies the economic activity. Thus, this contribution aims to introduce a conceptual framework regarding «good living» to understand that the operational function of social welfare cannot be addressed in a unidimensional way, just considering the economy, but also environmental variables, such as public health, quality of life, social integration, access to social rights, among others. All this has an impact on the identification of indicators that also measure these variables, intending to allow all the people to be drivers in social, political, and economic development in a sustainable manner.
\end{abstract}

Keywords: "Buen vivir", social welfare, living well, economism of law, right to a healthy environment, sustainable development, ecosocialism.

\title{
1. Introducción
}

Tras el desmantelamiento del estado de bienestar, en la era de la globalización, cualquier estudio acerca de los procesos nacionales para la toma de decisiones en materia económica se topa con las tentaciones que enfrenta el Estado en el ámbito de la soberanía territorial y económica. Siguiendo las líneas de una ideología neoliberal o ultraliberal, aquel se enfrenta a una paradoja recurrente construida en torno a las incertidumbres que representa una separación de las actividades socioeconómicas entre el Estado y las grandes empresas privadas. Se exige, por tanto, una reflexión sobre las funciones y el papel del derecho frente al dogma del crecimiento.

Ciertamente, «el Estado es el único que posee los medios coercitivos político-militares para establecer un orden basado en la ley, el Gobierno es el único que dirige la política exterior, la política interior, la política educativa, etc. Las estrategias autárquicas tienen como objetivo desmantelar el conjunto de misiones que incumben al Estado a fin de privatizarlas para integrarlas en la economía global»${ }^{1}$. Actualmente, dichas estrategias para la privatización de

1 Ulrich Beck, Pouvoir et contre-pouvoir à l'ère de la mondialisation (Flammarion, 2003), 273. 
las misiones estatales van acompañadas por el complejo concepto de «economía social de mercado» o de la idea híbrida de «economía circular», que se subsume bajo la etiqueta de «buena gobernanza»². Estas tácticas discursivas, sin embargo, tienen muy poco en cuenta las cuestiones sociales y culturales.

\section{La economicización del discurso en un contexto de globalización mundial}

A partir del siglo XX, el compromiso social que había caracterizado al «capitalismo industrial» se desmorona. Este modelo se había organizado en torno a un equilibrio relativo entre capital y trabajo, basado, a su vez, en la regulación de las relaciones sociales mediante normas legales, organizadas según un control de la economía que se ejercía a través de la política ${ }^{3}$. Hasta ahora, la teoría del crecimiento solo usaba dos factores de producción: trabajo y capital. A principios del siglo XXI, en cambio, las trayectorias de las acciones económicas se fueron adaptando paulatinamente ${ }^{4}$ en torno al «ciclo de vida de los productos (bienes y servicios)». La aceptación fluctuante del modelo de desarrollo sostenible ha integrado cuestiones ambientales, en términos de riqueza o recursos naturales.

Desde la década de 1970, tras los conflictos en torno al precio del petróleo, y ante la escasez de recursos naturales, los Estados desarrollaron sus políticas bajo el prisma de la economía medioambiental, en lugar de basar sus políticas públicas en las alertas científicas y en la aplicación gradual de un derecho ambiental $^{5}$. Apelando a la fuerza del lenguaje de la economía, se ha recalado en el concepto de «capital natural», que abarca aquellos recursos utilizados por el ser humano para satisfacer algunas de sus necesidades, sin que por ello los diferentes Estados del mundo los hayan tenido formalmente en cuenta a lo largo de esos años. Así pues, antes de que el modelo de desarrollo sostenible

2 Advertencia: El objetivo de esta comunicación no es el de volver a revisar los procesos de toma de decisiones, sino el de señalar la irreflexión, omisiones, olvidos, negligencias que se han dado en estos mecanismos en relación con los factores y conceptos sociales.

3 Véase Vincent de Gaulejac y Fabienne Hanique, Le capitalisme paradoxant: un système qui rend fou (París: Seuil, 2015).

4 Lo que nos remite a una noción individualista de «consumo responsable» en el que los poderes públicos no se preocupan por las consecuencias sociales que conlleva.

5 Véase Bertrand Hamaide et al., «Croissance et environnement: la pensée et les faits», Reflets et perspectives de la vie économique, n..$^{\circ} 4$ (2012): 9 y ss. 
se arraigara en el discurso político y legal, los desastres ocurridos durante la década de 1980 (Bhopal en diciembre de 1984; Chernóbil en abril de 1986), la desaparición de muchas especies y el incremento de la contaminación han puesto en tela de juicio los equilibrios socioecológicos, pero no por ello han frenado la financiarización de la economía.

En consecuencia, durante la década de 1990:

[El] contexto de las relaciones entre medio ambiente y crecimiento económico y, más allá, entre medio ambiente y actividad económica [había] llevado a reconsiderar la cuestión de los instrumentos de las políticas e incentivos ambientales al privilegiar tres aspectos: una mayor y continua integración de las consideraciones ambientales en las decisiones de los agentes económicos; - el cambio en las trayectorias de desarrollo tecnológico y económico de largo plazo y una gestión de la dinámica de generación de posibilidades [...]; - la posibilidad de diseñar políticas y acciones que puedan servir a otros objetivos de la comunidad, además de los objetivos ambientales, y en particular considerando las prioridades del momento... ${ }^{6}$.

Los discursos pronunciados en ese momento incluyeron una «disociación entre el capitalismo financiero desterritorializado y el real ${ }^{7}$, asegurando, independientemente de las fronteras nacionales, los instrumentos de optimización fiscal.

La era de la globalización amaneció de manera flagrante.

A pesar de las reivindicaciones sociales y las reticencias de la población frente a las consecuencias de la mercantilización excesiva de bienes y servicios, los postulados rectores de la década del 2000 validaron las medidas gubernamentales «promoviendo la jubilación financiada, el recurso a seguros privados, el desarrollo de la participación accionarial de los empleados, la privatización de los servicios públicos, la tolerancia de los sistemas de exención de impuestos, la aceptación de las normas internacionales de contabilidad diseñadas por organizaciones privadas» ${ }^{8}$.

Siguiendo este movimiento, las políticas públicas se han desjudicializado y rendido ante los análisis económicos; con ello se han sometido, a su vez, a las opiniones y recomendaciones de las agencias de calificación que evalúan

6 Olivier Godard y Olivier Beaumais, «Économie, croissance et environnement: de nouvelles stratégies pour de nouvelles relations», Revue économique 44, n. ${ }^{\circ} 1$ (1993): 146.

7 Gaulejac y Hanique, Le capitalisme paradoxant, 55.

8 Gaulejac y Hanique, Le capitalisme paradoxant, 57. 
—según sus estándares particulares y con sus propios métodos contables (imbuidos, por cierto, de una ideología profundamente liberal) — el atractivo de cada país. Este método de atracción, confeccionado para los inversores (empresas multinacionales), no se calcula recurriendo a parámetros sociales y ambientales, que combinan calidad de vida, salud, trabajo, ocio, medios de vida apropiados ${ }^{9}$, relativos tanto a los derechos fundamentales esenciales como al bienestar social.

Asimismo, junto con la problemática del desarrollo sostenible, los atributos del crecimiento económico impulsados por las aprensiones consumistas debían ser rediseñados en relación con las poblaciones ${ }^{10}$ y los entornos, fuesen los que fuesen. Por tanto, las inquietudes ambientales aparecerían como «elementos estratégicos de la competitividad económica de empresas y naciones» ${ }^{11}$.

La gestión de los recursos naturales del planeta se está volviendo crucial; estos, sin embargo, todavía son considerados riqueza, por lo que los lenguajes del mercado han interferido en su situación. Al respecto, Hamaide et al. sostienen: «... la idea de mantener el stock de capital natural requiere que el uso de los recursos naturales renovables no supere su tasa de renovación, que los recursos agotables se extraigan a un ritmo que permita su sustitución por recursos renovables y que las emisiones de residuos estén por debajo de la capacidad de asimilación del medio ambiente. Algunos van un paso más allá y creen que los servicios ambientales o los ecosistemas pueden ser únicos e irreemplazables, lo que requeriría mantener un umbral mínimo de este capital natural en particular» ${ }^{12}$.

9 Por ejemplo, en Francia, desde el Preámbulo de la Constitución de 1946 se integran a normas constitucionales: «el derecho a obtener un empleo»; la participación del trabajador, "por intermedio de sus delegados, a la determinación colectiva de sus condiciones de trabajo así como a la gestión de las empresas»; la garantía de «la protección de la salud, la seguridad material, el descanso y el ocio»; el «derecho de obtener de la colectividad los medios convenientes de existencia». En el Perú, la Constitución de 1993 dice en su art. 2. ${ }^{\circ}$ : «... Toda persona tiene derecho: [...] 15. A trabajar libremente, con sujeción a ley» [...]; «22. A la paz, a la tranquilidad, al disfrute del tiempo libre y al descanso, así como a gozar de un ambiente equilibrado y adecuado al desarrollo de su vida»; asimismo, el art. 22 anota que: «El trabajo es [...] base del bienestar social y un medio de realización de la persona»; el art. 24 señala que: «El trabajador tiene derecho a una remuneración equitativa y suficiente, que procure, para él y su familia, el bienestar material y espiritual».

10 Véase Cornelius Castoriadis, La montée de l'insignifiance, t. 4: Les carrefours du labyrinthe (Seuil, 1996).

11 Godard y Beaumais, «Économie, croissance et environnement», 144.

12 Hamaide et al., «Croissance et environnement», 15. 
Estos enfoques no pueden ignorar las relaciones sociales ni la necesidad de crear vínculos entre las actividades, entre las comunidades y entre los individuos.

Tal debe ser el eje rector del derecho en las decisiones que tomen los poderes públicos en materia económica.

La noción de buen gobierno, por tanto, se construyó junto con la retórica jurídica; en la actualidad, no obstante, forma parte de los arcanos de la protección ambiental internacional. Esto implica la existencia de un sistema legal que exige el respeto al medio ambiente, sin que necesariamente implique la existencia de una relación directa con las cuestiones sociales.

Pero el derecho a un medio ambiente sano no se entiende en términos genéricos: se percibe como un derecho humano fundamental, aunque, por su propia naturaleza, sea colectivo, común. Alexandre Kiss sitúa su fundamento en la declaración adoptada por la Conferencia de las Naciones Unidas sobre el Medio ambiente Humano ${ }^{13}$ (celebrada en junio de 1972 en Estocolmo), cuyo primer principio es: «El hombre tiene el derecho fundamental a la libertad, la igualdad y a condiciones de vida satisfactorias, en un entorno cuya calidad le permita vivir con dignidad y bienestar. Tiene el deber solemne de proteger y mejorar el medio ambiente para las generaciones presentes y futuras».

Por su parte, el párrafo 1 del artículo 4 del Convenio 169 de la Organización Internacional del Trabajo sobre los Pueblos Indígenas y Tribales en los Países Independientes (1989), ampliando el terreno en el que se basa el mencionado derecho, invita a los Estados a tomar acciones que salvaguarden el medio ambiente de estos pueblos: «Deben adoptarse medidas especiales, según sea necesario, con el fin de salvaguardar las personas, las instituciones, los bienes, el trabajo, la cultura y el medio ambiente de los pueblos interesados». A esto se suma el artículo 24 de la Carta Africana de Derechos Humanos y de los Pueblos (1981), que proclama: «Todos los pueblos tienen derecho a un entorno satisfactorio y mundial propicio para su desarrollo» ${ }^{14}$.

Como resultado de los citados documentos, se hace necesario abrir nuevas perspectivas en lo que respecta a los derechos de los pueblos autóctonos. El artículo 25 de la Declaración de las Naciones Unidas sobre los Derechos

13 Alexandre Kiss, «Environnement, droit international, droits fondamentaux», Cahiers du Conseil constitutionnel, n. ${ }^{\circ} 15$ (2004), https://www.conseil-constitutionnel.fr/nouveaux-cahiers-duconseil- constitutionnel/environnement-droit-international-droits-fondamentaux

14 Kiss, «Environnement, droit international, droits fondamentaux». 
de los Pueblos Indígenas (13 de septiembre de 2007) establece: «Los pueblos indígenas tienen derecho a mantener y fortalecer sus vínculos espirituales especiales con las tierras, los territorios, las aguas fluviales y costeras y otros recursos que tradicionalmente ocupan y utilizan, y a asumir sus responsabilidades a este respecto con las generaciones futuras».

En Francia, la fórmula paradójica del artículo 6 de la Carta Ambiental de 2004 resume el enfoque del desarrollo económico sostenible: «Las políticas públicas deben promover el desarrollo sostenible. Para ello, concilian la protección y el desarrollo del medio ambiente, el desarrollo económico y el progreso social» ${ }^{15}$.

Las proyecciones económicas ya no pueden desarrollarse independientemente de los entornos naturales, culturales y sociales. Por este motivo, se lleva a los gobiernos a hacer un balance de dichos entornos y a responder a la necesidad de reconocimiento que tienen los pueblos, así como de las demandas sociales.

Surgía una preconfiguración del bienestar (buen vivir) más que del vivir bien.

\section{Reconsideración de las relaciones entre los Estados y las sociedades civiles}

Cuestionar el respeto del medio ambiente, la tierra, el agua, la naturaleza, la flora, la fauna, etc., está vinculado a las características que presentan las relaciones entre el Estado y la sociedad civil, entre el Estado y los pueblos indígenas. El modelo de desarrollo sostenible inducido para el necesario apaciguamiento de las relaciones sociales se enfrenta, así, al que impone la dinámica competitiva del mercado. Para Jean-Luc Dubois y François-Régis Mahieu: «[son tres los criterios] constitutivos de la sostenibilidad: la accesibilidad de todas las personas a los bienes y servicios, el fortalecimiento de las capacidades de todo tipo y la equidad frente a todos los recursos disponibles y transmisibles. El concepto de sostenibilidad del desarrollo implica, basándose en

15 Ley constitucional n. ${ }^{\circ}$ 2005-205 del 1 de marzo de 2005 relativa a la Carta Ambiental, JORF, 2 de marzo de 2005, p. 3697. 
estos tres criterios, una progresión constante con la preservación de los beneficios en términos de satisfacción de las necesidades y crear capacidades» ${ }^{16}$.

Este modelo de desarrollo se concibe, por lo tanto, a expensas de comprender el concepto de conexión social. Bouvier señala las conmociones causadas por el hiperindividualismo:

La pérdida del nexo social se refleja en el énfasis puesto en el éxito individual, que se traduce en inclusión, salario, consumo y satisfacción de las necesidades materiales. El Estado, es decir, la institución que representa la organización social presta poca consideración a aquellos que no encajan individualmente en este esquema de posicionamiento esencialmente personal. [...]. Su acción [del Estado] se lleva a cabo principalmente a través de un aparato de leyes y mecanismos legales y policiales para asegurar un statu quo. Se trata principalmente de hacerse cargo de la tranquilidad de los propietarios y que circulan riqueza ${ }^{17}$.

El desglose del vínculo social y la pérdida de atención al equilibrio de las relaciones sociales pueden discernirse en la composición jurídica de una categoría específica de personas reunidas según el criterio de exclusión.

Pierre Bouvier se refiere a un «estado de los excluidos», un estatus que «significa la externalización del potencial de satisfacción relacionado con el empleo y, por lo tanto, con el salario y los logros del Estado de bienestar. Este no permite, debido a la falta de medios económicos, el acceso a los bienes y paradigmas de la sociedad de consumo. El término resume y dibuja la ruptura de las normas sociales y lo que esto implica como consecuencia: el aislamiento, el distanciamiento, así como el término indica, una negación de pertenencia al modelo social efectivo ${ }^{18}$.

Sin embargo, en una configuración centrada en el desarrollo económico, incluso si es sostenible, como señalan Jean-Luc Dubois y François-Regis Mahieu, «el aumento de la desigualdad, cualquiera que sea su origen - crecimiento económico, reducción de la pobreza específica o protección del medio ambiente- genera tensiones sociales, explica en parte el aumento de la violencia y puede conducir a graves desequilibrios sociales» ${ }^{19}$.

16 Jean-Luc Dubois y François-Régis Mahieu, «La dimension sociale du développement durable: réduction de la pauvreté ou durabilité sociale?», en Développement durable? Doctrines, pratiques, évaluations, ed. Jean-Yves Martin (Éditions IRD, 2002), 75.

17 Pierre Bouvier, Le lien social (Gallimard, coll. Folio Essais, 2005), 250.

18 Bouvier, Le lien social, 251.

19 Dubois y Mahieu, «La dimension sociale du développement durable», 78. 
El resultado es la necesidad de revisar las relaciones entre el Estado y la sociedad civil a la luz de la Ley, sin centrarse en la exploración infinita del enriquecimiento económico. Pero los Estados, ante la dificultad de poner de relieve los vínculos entre el Estado y la sociedad civil, invierten en formas de relaciones que consolidan las dinámicas clasificatorias de diversidad social y cultural, en lugar de exponer las expectativas inherentes a la composición del vínculo social. Como señala Jean-Marc Callois:

... el término «relaciones sociales» es extremadamente polisémico. Abarca una multitud de relaciones, incluyendo, por ejemplo, relaciones cliente-proveedor, relaciones jerárquicas o de equipo dentro de una organización, relaciones entre competidores en la misma rama, relaciones entre consumidores que influyen en las elecciones de cada uno, o relaciones entre emprendedores y responsables políticos [...]. Estas relaciones pueden considerarse de naturaleza institucional, al igual que la noción de relaciones salariales en la teoría de la regulación. Pero la literatura sobre el capital social sostiene que los factores sociales en su conjunto pueden tener un impacto en los fenómenos económicos. Así, por ejemplo, la existencia de una identidad local sólida, heredada de un pasado lejano, puede tener efectos positivos en los incentivos económicos actuales ${ }^{20}$.

Bajo la presión del culto al mercado, surgió el término capital social, como algo distinto de relaciones sociales; vincula otra forma de abordar las cuestiones económicas mediante la integración de determinaciones demográficas, geográficas, biológicas y ecológicas. La incorporación del concepto de capital social por los economistas transforma los presupuestos sociológicos, pues tiende a crear una idea de las relaciones sociales en torno a la obtención de bienes que generan beneficios. Las propias palabras trazan la intención de insertar el estudio de pretendidos nuevos modos de desarrollo desde una perspectiva capitalista: «Tener capital social es, por lo tanto, ser capaz de movilizar las relaciones sociales en su beneficio» ${ }^{21}$.

La preponderancia del vocabulario económico indica una investigación particular por separar el ámbito económico del ámbito social, a menudo en beneficio del primero, al tiempo que pretende incorporar preocupaciones ambientales o ecológicas sin movilizar los aspectos culturales consustanciales a

20 Jean-Marc Callois, «Capital social et développement économique local: pour une application aux espaces ruraux français ", Revue d'Économie régionale E urbaine, octubre, n. . 4, (2004): 554.

21 Callois, «Capital social et développement économique local», 554. 
las presunciones de diversidad cultural señaladas en el artículo 3 de la Declaración Universal de la Unesco sobre la Diversidad Cultural (2 de noviembre de 2001): «La diversidad cultural amplía las opciones que se le ofrece a cada uno; es una de las fuentes de desarrollo, entendidas no solo en términos de crecimiento económico, sino también como un medio de acceso a una existencia intelectual, emocional, moral y espiritual satisfactoria».

La disyunción entre lo social y lo económico es inherente a los discursos neoliberales que se apartan de las líneas trazadas por los principios de derecho; «complementa la negativa a imaginar otra economía mixta. El resultado del descrédito público y la victoria del sector privado es un signo de impotencia» ${ }^{22}$.

El pretexto falso de una imbricación entre lo cultural, lo social y lo económico penetra en los discursos y siembra la confusión en las áreas del derecho público y privado, especialmente en los países que se rigen por el derecho romano. Estas amalgamas conducen a una degradación de la figura del Estado y otorgan a las fuerzas económicas un poder autónomo.

Como la legitimidad de cualquier Estado se basa en la sintaxis del interés general -y necesariamente en los intereses privados-, tan pronto como las actividades $\mathrm{u}$ operaciones estatales se someten a las normas comerciales o a una ley de competencia modificada por los acuerdos entre multinacionales, aquel se embarca en un proceso de reescritura económica del derecho ${ }^{23}$, esencialmente sobre la base de normas de obediencia privada (convenios colectivos, acuerdos de asociación, etc.).

Sin embargo, ante el deterioro del estado del planeta, asistimos a un aumento de las expectativas individuales - a menudo consumistas - , a un aumento en la intensidad de los desafíos sociales, a la acentuación de las demandas socioculturales inducidas por la instrumentalización de la noción de diversidad. Estos factores han obligado a las fuerzas dominantes a reconstituir conceptos jurídicos que sirvan para revisar ciertos aspectos de las políticas públicas implementadas, con la intención declarada, aunque ilusoria, de satisfacer las demandas individuales, las aspiraciones sociales y los proyectos socioculturales «al mismo tiempo».

22 Philippe Herzog, «La progression du libéralisme social», en L'Europe après l'Europe: Les voies d'une métamorphose, dir. Philippe Herzog (De Boeck Supérieur, 2002), 107.

23 Véase Jacques Caillosse, L'État du droit administratif, collection Droit et Société (París: LGDJ, 2015); Jacques Caillosse, La constitution imaginaire de l'administration, collection Les voies du droit (PUF, 2008). 
Esta perspectiva, sin embargo, no tiende a desafiar las parábolas sistémicas de este liberalismo económico desenfrenado.

El derecho aparecería entonces como un instrumento artificial para conciliar el bienestar y el buen vivir, a pesar de que la decisión en materia económica, que sigue siendo una cuestión de derecho, tiene poco interés en estos factores sociales. Y es que «la teoría económica ha planteado el problema del Estado a imagen de la del mercado» ${ }^{24}$.

[En este sistema] el Estado intervendrá en interés de sus administrados cada vez que se produzcan fallos en el mercado, es decir, cada vez que las normas de funcionamiento del mercado no se respeten. En esta situación, el mercado está en una situación de debilidad, puesto que algunos bienes y servicios ya no serán producidos de manera óptima por él. En ese momento se pueden plantear intervenciones públicas para corregir el mercado, sea completando o brindando en su totalidad los bienes que ha dejado de proporcionar total o parcialmente. Es el caso de los bienes públicos puros, como la defensa Nacional, la Policía, etc., que tienen características tales que ningún productor privado tendría interés en producirlos, ya sea por las molestias, la contaminación o las desigualdades que su producción provocaría y que implican costes que el mercado no sabe tener en cuenta ${ }^{25}$.

Como resultado, los aspectos sociales, culturales y medioambientales, que están innegablemente asociados, difícilmente son tenidos en cuenta, ni por los Gobiernos ni por las empresas.

\section{Deserción del Estado económico: el olvido del buen vivir}

En efecto, «la idea de que en materia económica las personas públicas deben abstenerse de actuar $\mathrm{o}$, al menos, limitarse a las formas más indirectas y menos intrusivas de regulación en los mecanismos de mercado se ha visto claramente socavada en los últimos años» ${ }^{26}$. Se aprecian ya algunas advertencias, aunque sean implícitas.

24 Philippe Abecassis, «La résignation du décideur devant la décision collective», en La décision: une approche pluridisciplinaire des processus de choix, ed. Bénédicte Vidaillet (De Boeck Supérieur: 2005), 198.

25 Abecassis, «La résignation du décideur devant la décision collective», 199.

26 Jean-Marc Sauvé, Avant-propos, en Conseil d'État, Étude annuelle: L'action économique des personnes publiques (EDCE, 2015), 5. 
En cierto modo, ahora se trataría de frenar la sobredeterminación económica del derecho para reconocer formal y concretamente el contenido y el vigor de los derechos sociales. En estas circunstancias marcadas por las aspiraciones nacionales de desarrollo económico « los derechos sociales son esencialmente derechos de reclamación característicos de lo que la ciudadanía puede reclamar a las autoridades en términos de bienes y servicios económicos en tiempos difíciles y en las condiciones propias en las que estas pueden expresarse. Por lo tanto, proporcionan acceso a bienes y servicios que se prestan fuera del mercado y que no están directamente determinados por el comercio en el mercado... ${ }^{27}$.

Sin embargo, en términos de «relaciones sociales», es esencial superar la figuración individual que significa la noción de «ciudadanía social [que se definiría] en términos de derechos y deberes establecidos por la ley» ${ }^{28}$.

Entonces, surgen dos expectativas sociales y de la sociedad (comunes y colectivas): bienestar social y buen vivir, dos configuraciones que las decisiones tomadas en materia económica descuidan.

\section{1. Sobre el bienestar}

Generalmente entendido más allá del concepto central del concepto de servicio público, «satisfacer las necesidades colectivas» ${ }^{29}$, el bienestar (bienestar social) se refiere a los elementos que una persona siente que necesita para disfrutar de una buena calidad de vida. Sin embargo, "para alcanzar un cierto nivel de bienestar, uno debe ser capaz de "funcionar" adecuadamente (es decir, "hacer y ser"), o ser capaz de alcanzar una serie de objetivos: desplazarse, habitar, gozar de buena salud, alimentarse de forma equilibrada, tener un reconocimiento social y ser respetado, poder participar en decisiones colectivas,

27 Walter Korpi, «Un État-providence contesté et fragmenté. Le développement de la citoyenneté sociale en France. Comparaisons avec la Belgique, l'Allemagne, l'Italie et la Suède», RFSP 45, n. ${ }^{\circ} 4,(1995): 633$.

28 Korpi, «Un État-providence contesté et fragmenté», 633.

29 Según Jean-Luc Dubois y François-Régis Mahieu: ... la «satisfacción de las necesidades» comprende "fuera del consumo de bienes y servicios, el conjunto de bienes primarios y derechos a los cuales aspiran las personas, que se trate de la participación en las decisiones de política, del deseo de las relaciones sociales, de la predisposición a donar, de la vista de un bello paisaje, etc. Ello permite lograr un cierto nivel de bienestar». "La dimension sociale du développement durable: Réduction de la pauvreté ou durabilité sociale?», en Développement durable? Doctrines, pratiques, évaluations, ed. Jean-Yves Martin (Éditions IRD, 2002), 74. 
etc. Estas metas solo pueden alcanzarse si uno tiene las capacidades de "hacer" cosas y lograr determinadas "formas de vivir" $\gg^{30}$.

Si este conjunto se basa en la idea de "capacidades", inculcado a través de la propuesta de igualdad de oportunidades ${ }^{31}$, esta sigue dependiendo de la situación de la persona en sí misma. Las implicaciones, sin embargo, se corresponden con las representaciones del bienestar social:

El reforzar las capacidades de una persona aumenta sus posibilidades de realización y le permite mejorar su bienestar general. Visto desde fuera, esto se manifiesta en una mejora de su nivel de vida (a través de un trabajo que le proporcione ingresos), una mejora en sus condiciones de vida (seguridad alimentaria, vivienda, agua potable, salud, educación, etc.), el aumento de sus recursos (tierra y equipamiento, microcrédito, capital humano, etc.) y el aumento de sus otros potenciales (fortalecimiento de los lazos sociales, participación en el ejercicio del poder, modo de expresión cultural, creencias, etc.) ${ }^{32}$.

La función operativa del bienestar social no puede abordarse únicamente a nivel individual. Esta noción de bienestar social es multidimensional y concierne tanto a los pueblos como a su población. Moviliza varias causas combinadas con cuestiones económicas y medioambientales: salud pública (física y mental), medio ambiente saludable (calidad de vida) y equilibrio financiero (estándar de vida, integración social, acceso a los derechos sociales). Esto implica llevar a cabo evaluaciones en las que se exige la identificación de una serie de indicadores para medir el bienestar en relación con la situación socioeconómica del país: redistribución de impuestos/desigualdad de ingresos; clima/energía; comercio/producción; trabajo/empleo alfabetización/educación; salud/epidemiología; biodiversidad/gestión de los recursos; consumo/ reciclaje de residuos; etcétera. El objetivo es permitir que todas las personas puedan satisfacer sus necesidades y se beneficien de los derechos que les son propios y, al hacerlo, ser actores en el desarrollo social, político y económico.

Por lo tanto, el Estado debe proporcionar todas las condiciones necesarias para que cada individuo disfrute del mejor estado de salud posible, garantizándole el derecho al acceso a la atención sanitaria; igualmente, tiene que ga-

30 Dubois y Mahieu, «La dimension sociale du développement durable», 80.

31 Geneviève Koubi, «Véase l'égalité des chances: quelles chances en droit?», en L'égalité des chances. Analyses, évolutions, perspectives, Collection Recherches, eds. Geneviève Koubi y Gilles Guglielmi (La Découverte, 2000), 69 y ss.

32 Dubois y Mahieu, «La dimension sociale du développement durable», 80. 
rantizar un mínimo de recursos a todos los ciudadanos y permitirles acceder a condiciones satisfactorias que les permita llevar a cabo su trabajo, independientemente de las perspectivas económicas establecidas.

Sin embargo, en el discurso político o administrativo, bajo el pretexto del pragmatismo, existe una cierta «reticencia a captar la realidad social a través de percepciones subjetivas de los actores» ${ }^{33}$. Por lo tanto, más que tener en cuenta a la persona exclusivamente, se hace primordial volcarse también hacia las demandas colectivas, sociales y comunes. Así pues, otros factores no estrictamente económicos «pueden afectar el bienestar de la población: la calidad del entorno natural y cultural, la calidad de las relaciones sociales, las condiciones de trabajo, una buena o mala cobertura en el seguro relativo a los riesgos vitales (especialmente en temas de salud), etc. $\rangle^{34}$. Las cuestiones materiales no nos permiten ignorar los aspectos relacionales y sociales que interactúan. Por lo tanto, se ha observado que la «falta de correlación entre el crecimiento y el bienestar provienen del hecho de que el crecimiento económico conduce a unas mayores aspiraciones de las poblaciones, que se adaptan y se acostumbran a sus estándares: y a medida que algunos de estos deseos se satisfacen, otros aparecen ${ }^{35}$. Pero los proyectos de desarrollo económico a menudo están «lejos de beneficiar a todos los segmentos de la sociedad», a veces promueven «derivas autoritarias e intervencionistas» y no excluyen los «intentos de manipulación en el tablero de ajedrez geopolítico» a nivel internacional ${ }^{36}$.

Toda política social depende actualmente de los análisis económicos. Cualquier acción que se quiera emprender en materia social deriva de las decisiones económicas tomadas previamente.

Las decisiones que combinan el desarrollo económico y el progreso social pretenden inducir efectos redistributivos de la política económica, evitando la sobrevaloración de la propiedad privada, protegiendo a las personas de las alteraciones del mercado y de los cambios relacionados con la edad, desarrollando el potencial productivo de los miembros de la sociedad y asegurando el reparto de las consecuencias de las calamidades y cargas.

33 Rémy Pawin, «Le bien-être dans les sciences sociales: naissance et développement d'un champ de recherches», L'Année sociologique 64, n. ${ }^{\circ} 2$ (2014): 288.

34 Gérard Cornilleau, "Croissance économique et bien-être», Revue de l’OFCE 96, n. ${ }^{\circ} 1$ (2006): 12.

35 Pawin, «Le bien-être dans les sciences sociales», 280.

36 Véase Thandika Mkandawire, «Les politiques de développement social: un nouveau défi pour les sciences sociales», Revue internationale des sciences sociales 189, n. ${ }^{\circ} 3$ (2006): 427 y ss. 
Si bien el Estado se centra únicamente en la función distributiva, las medidas de política social no son sostenibles a largo plazo frente a las realidades demográficas y geográficas. En este caso, el Estado se vería privado de cualquier legitimidad; a mediano o a largo plazo se produciría una implosión política: «Un estado puramente distributivo fracasaría, porque no tendría ni los medios materiales de su política ni el apoyo político de las clases medias. Este es el destino de todos los regímenes "populistas", cuya política exclusivamente distributiva ha llevado a menudo a la inflación y al estancamiento, dejando a los pobres aún más pobres que antes. En cuanto a un régimen puramente "protector", fracasaría en ambos niveles y sería condenado por la dinámica demográfica» ${ }^{37}$.

Las modalidades de redistribución de la riqueza debían basarse en una noción de economía del bienestar, aunque, inevitablemente, «cualquier redistribución va en contra de los intereses de ciertos individuos en beneficio de otros» ${ }^{38}$.

Por lo tanto, las "variables de bienestar colectivo", como la salud - dependientes de la protección del medio ambiente ${ }^{39}$ - y la educación - la alfabetización-, deben introducirse en los procesos de toma de decisiones económicas como uno de los pilares principales. El riesgo, sin embargo, es que «el objetivo de la intervención pública [ya no sería] hacer cumplir mejor las preferencias de los consumidores, sino imponer las opciones consideradas mejores para todos [...]. Técnicamente, esta transformación se traduce en primer lugar en un cambio de lenguaje: ya no hablaremos, para designar preferencias colectivas, de la función de utilidad colectiva, sino de la función de bienestar social o incluso de la función de preferencia del Estado» ${ }^{40}$.

Si esta es la desventaja de un contexto estatal de bienestar social que utiliza las normas del derecho, al menos este se podrá comparar con cualquier

37 Thandika Mkandawire, «Les politiques de développement social», 433.

38 Philippe Abecassis, «La résignation du décideur devant la décision collective», 202.

39 Véase Myriam Legendre, «Environnement et santé», Santé Publique 15, n. ${ }^{\circ} 3$ (2003): 291 y ss. «El culto creciente del materialismo ha sacrificado los valores de los bienes inmateriales, como las satisfacciones que procura el medioambiente (la naturaleza, la belleza, la cultura, la seguridad, la convivialidad) cada vez que están en conflicto con un bien material con valor comercial. Por ejemplo, la pureza del aire y del agua, la integridad biológica de la comida, el silencio, los espacios verdes se han sacrificado por la búsqueda del dinero; la política europea de transportes ha empujado a la hipertrofia del transporte terrestre porque se consideró que las ventajas del tren en materia de seguridad y contaminación carecían de valor económico; el caso de la vaca loca muestra asimismo el peligro de haber sacrificado la salud en beneficio del lucro», p. 292.

40 Philippe Abecassis, «La résignation du décideur devant la décision collective», 196. 
sociedad democrática en la medida en que la protección de los derechos humanos y las libertades fundamentales son la base principal.

\section{2. Sobre el buen vivir}

El posicionamiento que consagraría las funciones de bienestar en los procesos de toma de decisiones solo puede materializarse a condición de que el Estado escoja para sus decisiones mecanismos de distribución, redistribución, reparto, pero sin privilegiar las necesidades de una clase social determinada. Si el bienestar es también un vivir bien, será la combinación del bienestar social y de un bienestar en la sociedad lo que nos permitirá introducir en los mecanismos de toma de decisiones un enfoque razonado del buen vivir.

La expresión buen vivir ${ }^{41}$ se acerca a la noción "vivir juntos», muy usada en Francia con el fin de mantener las formas relacionales de pluralidad inherentes a las civilizaciones recompuestas tras el pensamiento de la integración social ${ }^{42}$, esto, con el fin de respetar los espacios naturales y contrarrestar la «divinización de la actividad económica»" Por lo tanto, también se basa en el buen vivir específico de las conceptualizaciones latinoamericanas de los pueblos indígenas, ya que conservan el principio de «vivir en armonía y en equilibrio; en armonía con los ciclos de la Madre Tierra, el cosmos, la vida y la historia, y los equilibrios con toda la existencia formada» ${ }^{44}$.

Frente al cambio climático, se anunciaría un cambio de paradigma en los procesos de toma de decisiones, incluso si «el mundo occidental parece [...] hermético a los valores indígenas», ya que los occidentales «preocupados por

41 Véase Jean Ortiz, «Le concept andin de "buen vivir" et "1'écosocialisme"», Le Grand soir, 18 de septiembre de 2013, https:/ / www.legrandsoir.info/le-concept-andin-de-buen-vivir-et-l-ecosocialisme.html. A pesar de la dificultad de las traducciones: "La expresión "buen vivir" no podría ser traducida en francés por "bien être" ("bienestar"), "vivre bien" ("vivir bien"), "vivre mieux" ("vivir mejor"), sino más bien por: la vie belle ("la vida bella"), la vie bonne ("la buena vida"), les jours heureux ("los días felices"), que dicho sea de paso era el título del Programa del Consejo Nacional de la Resistencia luego de la Segunda Guerra Mundial. Con el "buen vivir", la felicidad está en la revolución, en el deneoliberalismo, la desmercantilización...». Sin embargo, "en Francia, el "vivir bien" tiene otro contenido; significa ver sus necesidades físicas materiales, intelectuales, satisfechas cuantitativamente, no tener problemas financieros, poder consumir sin restricciones, sin privación, sin frustración, vivir a gusto y sin problemas materiales».

42 Véase Carlos González Palacios, «La pensée sociale andine: une alternative au modèle occidental d'inclusion sociale», en Estrategias de consolidación de las entidades públicas, eds. Carlos Gonzáles Palacios, Thilo Rensmann y Lionel Zevounou (Lima: Centro de Altos Estudios Nacionales, 2015), 133 y ss.

43 Véase Alberto Acosta, Le buen vivir: pour imaginer d'autres mondes (Utopia, 2014).

44 Julien Vanhulst y Adrian E. Beling, «Buen vivir et développement durable: rupture ou continuité ?», Écologie E politique 46, n. 1 (2013): 44. 
"vivir mejor", no entienden por qué los pueblos indígenas como los de las dos Américas quieren "vivir bien" $\gg^{45}$.

Pero en esta oposición entre vivir mejor y vivir bien, los estados difícilmente se deshacen de las exigencias económicas que ellos mismos han creado. Se obtiene un «nuevo mapeo de conflictos provocados por la movilización en torno a temas ambientales, liderados por organizaciones indígenas y campesinas, así como nuevos movimientos socioambientales ${ }^{46}$.

La distancia entre las normas de comportamiento vivir bien y vivir mejor es innegable, pero también hay una distancia entre vivir bien y buen vivir.

Vivir bien implica una atención sostenida al medio ambiente, a los recursos naturales; vivir mejor se entiende como «sinónimo de individualismo, desinterés en los demás, búsqueda de beneficios, de ahí una necesaria explotación de las personas y de la naturaleza. "Vivir mejor" también significa un progreso ilimitado, acumulación de bienes materiales, competencia, búsqueda de energía, consumo excesivo. Todo esto crea una sociedad desigual, desequilibrada, depredadora, antropocéntrica y contra la naturaleza ${ }^{47}$.

Señalando los planes de desarrollo como fuentes en la introducción de los conceptos de vivir bien y buen vivir en las constituciones boliviana y ecuatoriana, Alfredo Gómez-Muller confirma que «la diferencia entre el vivir mejor, que se interpreta como un modo de vida individualista y "occidental" donde cada individuo vive "separado de los demás o incluso a expensas de los demás, y separado de la naturaleza", el buen-vivir expresa "esencialmente valores humanos" que, como la "ayuda mutua y la solidaridad", deben sustentar el modelo de desarrollo de la sociedad $»^{48}$.

Si bien existen correlaciones entre el vivir bien y el buen vivir, el primero se elabora aún en términos individualistas, abogando por la correspondencia, la reciprocidad y la solidaridad, mientras que el concepto buen vivir se despliega esencialmente en términos sociales y culturales, haciendo hincapié en la convivencia, la interdependencia y la armonía. El buen-vivir supera el vivir-bien;

45 Françoise Morin, «Les droits de la Terre-Mère et le bien-vivre, ou les apports des peuples autochtones face à la détérioration de la planète» 42, n. 2 (2013): 332. Vale anotar que «las ideas transmitidas por este concepto han nutrido la elaboración de marcos normativos, como las nuevas constituciones andinas: la de Ecuador, adoptada en 2008, la de Bolivia en 2009», 334.

46 Maristella Svampa, «Néo-"développementisme" extractiviste, gouvernements et mouvements sociaux en Amérique latine», Problèmes d'Amérique latine 81, n. 3 (2011): 104.

47 Morin, "Les droits de la Terre-Mère et le bien-vivre», 332.

48 Alfredo Gómez-Müller, «Le Buen vivir: une critique andine de la modernité capitaliste», Contretemps, 14 de marzo de 2019, https: / / www.contretemps.eu/buen-vivir-critique-andinemodernite- capitaliste/ 
por lo tanto, es un medio de resistencia a la mercantilización de la tierra y de los bienes comunes, así como la lucha contra la explotación económica de los recursos y las personas; constituye, por lo tanto, un mecanismo para combatir las actividades de apropiación de productos naturales con el fin de destinarlos a la comercialización. En muchos estados, sin embargo, en la medida en que persisten los vínculos entre el extractivismo y el neoliberalismo, las expectativas que conforman el vivir bien y el buen vivir parecen estar dislocadas: «En el contexto actual, el extractivismo debe entenderse como un modelo de acumulación basado en la sobreexplotación de los recursos naturales en gran medida no renovables y en el desplazamiento de las fronteras de los territorios hasta ahora considerados "improductivos". Por último, este modelo incluye no solo actividades estrictamente extractivas (sector minero y petrolero) sino también otras actividades (como la agroindustria y los biocombustibles) que fomentan la lógica extractiva mediante la consolidación de la monoproducción ${ }^{49}$.

Las perspectivas de una institución del modelo alternativo de buen vivir nos invitan a «pensar en una nueva economía de necesidades, más justa, más racional, más eficiente energéticamente, menos contaminante, menos modos de desarrollo "extractivistas" ». ${ }^{50}$ Estos, sin embargo, deben ser matizados, ya que, además de la distancia que existe entre el vivir bien y el buen vivir, hay una instrumentalización política y económica de estas dos concepciones del mundo, de la tierra y de la vida en la sociedad.

La noción de vivir bien es plural en su materialidad, pero se ha desvinculado de la espiritualidad cultural del pensamiento autóctono (o según las marcas lingüísticas, indígenas) "para convertirse en un verdadero concepto autónomo. El vivir bien tiene una dimensión reactiva, que se opone a las nociones de modernidad y desarrollo, pero también una dimensión proactiva, que propone una refundación ontológica del derecho y la política. En su dimensión reactiva, el vivir bien se caracteriza en parte por su oposición a la modernidad occidental y al dualismo cartesiano» ${ }^{51}$.

49 Svampa, "Néo-"développementisme" extractiviste, gouvernements et mouvements sociaux, 105.

50 Ortiz, «Le concept andin de "buen vivir" et "l'écosocialisme"».

51 Victor Audubert, "La notion de Vivir Bien en Bolivie et en Équateur, réelle alternative au paradigme de la modernité ?», Cahiers des Amériques latines, n. ${ }^{\circ} 85$ (2017): 94. 
Las concepciones del buen vivir combinan los dos lados de una proyección social, en la que la cultura y la naturaleza ${ }^{52}$ están indefectiblemente unidas. Las construcciones sociales resultantes se basan en un enfoque basado en los recursos naturales.

En muchas partes del mundo, la protección de la naturaleza continúa siendo uno de los parámetros excluidos de las estimaciones en las que se basan las decisiones económicas. No todos los Estados adoptan el patrón internacionalista de bienes públicos globales —o bienes comunes naturalesya que la dinámica del progreso económico se trabaja en torno a la soberanía del Estado y a la propiedad pública o privada. Por lo tanto, la lucha por la protección de los recursos naturales parece limitarse a territorios marcados. Sin embargo, la noción del bien público mundial «transcribe la creciente preocupación por los problemas mundiales como el cambio climático, la inseguridad, el desarrollo de pandemias mundiales como el VIH/sida, etc., en un contexto de globalización de las economías» ${ }^{53}$. Se cuestionan aquellos principios jurídicos basados en el concepto de «propiedad» con el fin de repensar las relaciones económicas y sociales dentro del modelo de desarrollo sostenible, más allá de las estadísticas y balanzas comerciales. Así pues, «el buen vivir ya se está afirmando como un discurso crítico a la ideología del progreso, la racionalización y el universalismo específico de la modernidad europea. En este sentido, el discurso del buen vivir aporta una nueva perspectiva a los retos del desarrollo sostenible, el último avatar del discurso sobre el desarrollo, que ha tratado de amortiguar las críticas al desarrollo en el terreno económico, social y ambiental» ${ }^{54}$.

Sin recurrir directamente al modelo de la economía social y solidaria que abarca el desarrollo agrario, la educación, el desarrollo social y la lucha contra el hambre, el trabajo y el empleo, etc., el problema de la «ecologizacion de la

52 El artículo 276 de la Constitución Ecuatoriana «evoca uno de los objetivos del Buen Vivir: "recuperar y conservar la naturaleza y mantener un medioambiente sano y sostenible" $\mathrm{e}$ igualmente garantizar el acceso y la calidad del agua, el aire, los suelos y los excedentes de los recursos naturales (citado por Víctor Audubert, «La notion de Vivir Bien en Bolivie et en Équateur?, 99).

53 Jérôme Ballet, «Propriété, biens publics mondiaux, bien(s) commun(s): une lecture des concepts économiques», Développement durable et territoires, n. 10 (2008), https: / /journals.openedition.org/developpementdurable/5553\#text.

54 Vanhulst y Beling, «Buen vivir et développement durable, 43. 
sociedad $»^{55}$ se vuelve irresistible para hacer frente a la «degradación ambiental, el agotamiento de los recursos naturales, el progreso de la desertificación, el aumento de las disparidades globales con una creciente división de entre ricos y pobres» ${ }^{56}$.

Esta apertura tiende a alterar el orden jurídico establecido en los preceptos de la globalización económica. Si el buen vivir ofrece «otra visión del mundo» ${ }^{57}$, solo podrá «ser una alternativa al desarrollo capitalista ${ }^{58}$ a condición de que no sea decidido por las instancias gubernamentales. Y esto debido a que el «"buen vivir" no se decreta desde arriba»" sociedad, en las comunidades, siguiendo un principio participativo social.

Pero la esperanza de armonía entre la población, la naturaleza y la cultura, intrínseca al concepto de buen vivir, se está apagando. Porque:

... en paralelo con el creciente uso de este concepto en el discurso político, ya venimos asistiendo desde hace algunos años al cuestionamiento del vivir bien por parte de aquellos mismos gobiernos que contribuyeron al desarrollo de las nuevas constituciones, hasta el punto de ver esta noción transformada y desvestida de su significado original. De hecho, el vivir bien parece estar ahora al servicio de una economía neoextractivista y desarrollista, en oposición a una verdadera política de promoción de los derechos de los pueblos indígenas y la protección de la naturaleza. Por lo tanto, se puede cuestionar la evolución del significado otorgado a este concepto original, pero también a los intentos de integrarlo en diferentes legislaciones andinas, así como las tensiones que surgen de la aplicación legal de este concepto y que conducen al surgimiento de una verdadera «paradoja del vivir bien ${ }^{60}$.

Sin embargo, es difícil ignorar que «una de las consecuencias del impulso extractivista actual es la proliferación de conflictos sociales en torno a cuestiones ambientales. Esto se refleja en el auge de las luchas ancestrales por la

55 Frase de Fabrice Flipo, «Pour des droits de la Nature», Mouvements 70, n. 2 (2012): 122 y ss.

56 Morin, «Les droits de la Terre-Mère et le bien-vivre», 326 (con referencia al discurso de Kofi Anan en la Cumbre Mundial sobre Desarrollo Sostenible, Johannesburgo, 2 de septiembre de 2002: www.un.org/events/wssd/statements/sgF.htm.

57 Tomando el título del artículo de Pablo Solón, «Le "buen vivir", une autre vision du monde» (trad. del español por Jean Merckaert), Revue Projet, 362, n. ${ }^{\circ} 1$ (2018): 66 y ss.

58 Morin, «Les droits de la Terre-Mère et le bien-vivre», 337.

59 Título de uno de los párrafos del artículo de Pablo Solón, «Le "buen vivir", une autre vision du monde», 71 .

60 Audubert, «La notion de Vivir Bien en Bolivie et en Équateur, 92. 
tierra de los movimientos indígenas y campesinos, así como en el surgimiento de nuevas formas de movilización y participación ciudadana en torno a la defensa de los recursos naturales (definidos como "bienes comunes"), la biodiversidad y el medio ambiente» ${ }^{61}$.

Como señala Víctor Audubert, "ya sea el vivir bien o el buen vivir, se puede observar que estas nociones están al servicio de un cierto paradigma, el del desarrollo de una cierta ontología, la de la modernidad, y de una cierta economía, la del capitalismo. En este contexto, la libre determinación política y económica de las comunidades indígenas parece ilusoria, y la concesión de derechos a la naturaleza parece tener que permanecer en el estadio de utopía ${ }^{62}$.

Sin embargo, el ecosocialismo implica «la propiedad colectiva de los principales medios de producción, la planificación democrática y ecológica, el control de las prioridades económicas y sociales del pueblo, las decisiones de inversión, la reorganización del modo de producción sobre la base de las necesidades y los valores de uso reales, la reorientación de la política energética, el transporte... ». El ecosocialismo a menudo se asocia con el buen vivir, «incluye tanto el pensamiento crítico como una estrategia de lucha. ¿Cómo podemos ir más allá del modo dominante, dañino y en constante expansión de producción y consumo? El ecosocialismo es, por el momento, más una investigación que un modelo exitoso. Parte de la constatación de una «crisis de la civilización» inducida por el capitalismo financiero; este último transforma todo en mercancía, y su carrera para lograr las tasas de beneficio más altas posibles, la acumulación ilimitada de capital resulta destructiva no solo para los seres humanos, sino también para todos los ecosistemas ${ }^{63}$.

En estos tiempos difíciles que nos acontecen, se requiere una refundación de los paradigmas económicos, y esto tal vez se logre centrando la atención tanto en la población en general como en los pueblos indígenas. No obstante, no parece ser este el horizonte hacia el que tiende la «buena gobernanza».

61 Svampa, «Néo-"développementisme” extractiviste, gouvernements et mouvements sociaux», 106.

62 Audubert, «La notion de Vivir Bien en Bolivie et en Équateur», 104-105.

63 Ortiz, «Le concept andin de "buen vivir" et "l'écosocialisme"». 


\section{REFERENCIAS}

Abecassis, Philippe. «La résignation du décideur devant la décision collective». En $L a$ décision: une approche pluridisciplinaire des processus de choix, editado por Bénédicte Vidaillet. De Boeck Supérieur, 2005.

Acosta, Alberto. Le buen vivir: pour imaginer d'autres mondes. Utopia, 2014 (trad. del español por Marion Barailles: Buen Vivir Sumak Kawsay: una oportunidad para imaginar otros mundos. Quito: Abya Yala, 2012).

Audubert, Victor. «La notion de Vivir Bien en Bolivie et en Équateur, réelle alternative au paradigme de la modernité?». Cahiers des Amériques latines, n. ${ }^{\circ} 85$ (2017): 91-108.

Ballet, Jérôme. «Propriété, biens publics mondiaux, bien(s) commun(s): une lecture des concepts économiques». Développement durable et territoires, n. ${ }^{\circ} 10$ (2008). https:/ / journals.openedition.org/developpementdurable/5553\#text

Beck, Ulrich. Pouvoir et contre-pouvoir à l'ère de la mondialisation. Flammarion, 2003.

Bouvier, Pierre. Le lien social. Colección Folio Essais. París: Gallimard, 2005.

Caillosse, Jacques. La constitution imaginaire de l'administration. Colección Les voies du droit. Presses Universitaires de France (PUF), 2008.

Caillosse, Jacques. L'État du droit administratif. Colección Droit et Société. París: LGDJ, 2015.

Callois, Jean-Marc. «Capital social et développement économique local: pour une application aux espaces ruraux français». Revue d'Économie régionale E urbaine, octubre, n. ${ }^{\circ} 4$ (2004): 551-577.

Castoriadis, Cornelius. La montée de l'insignifiance, t. 4: Les carrefours du labyrinthe. Seuil, 1996.

Cornilleau, Gérard. "Croissance économique et bien-être». Revue de l'OFCE 96, n. ${ }^{\circ}$ (2006): 11-34. 
Dubois, Jean-Luc y François-Régis Mahieu. «La dimension sociale du développement durable: réduction de la pauvreté ou durabilité sociale?». En Développement durable ? Doctrines, pratiques, évaluations, editado por Jean-Yves Martin. Éditions IRD, 2002.

Flipo, Fabrice. «Pour des droits de la Nature». Mouvements 70, n. ${ }^{\circ}$ (2012): 122-137.

Gaulejac, Vincent de y Fabienne Hanique. Le capitalisme paradoxant: un système qui rend fou. París: Seuil, 2015.

Godard, Olivier y Olivier Beaumais. «Économie, croissance et environnement: de nouvelles stratégies pour de nouvelles relations». Revue économique 44, n. ${ }^{\circ} 1$ (1993): 143-176.

Gómez-Müller, Alfredo. «Le Buen vivir: une critique andine de la modernité capitaliste», Contretemps, 14 de marzo de 2019. https://www.contretemps.eu/buen-vivircritique-andine-modernite- capitaliste/

González Palacios, Carlos. «La pensée sociale andine: une alternative au modèle occidental d'inclusion sociale». En Estrategias de consolidación de las entidades públicas, editado por Carlos Gonzáles Palacios, Thilo Rensmann y Lionel Zevounou, 133148. Lima: Centro de Altos Estudios Nacionales, 2015.

Hamaide, Bertrand, Sylvie Faucheux, Martin Neve y Martin O'Connor. «Croissance et environnement: la pensée et les faits». Reflets et perspectives de la vie économique, $\mathrm{n}$. 4 (2012): 9-24.

Herzog, Philippe. «La progression du libéralisme social». En L'Europe après l'Europe: les voies d'une métamorphose, dirigido por Philippe Herzog. De Boeck Supérieur, 2002.

Kiss, Alexandre. «Environnement, droit international, droits fondamentaux». Cahiers $d u$ Conseil constitutionnel, n. ${ }^{\circ} 15$ (2004). https://www.conseil-constitutionnel. $\mathrm{fr} /$ nouveaux-cahiers-du-conseil-constitutionnel/environnement-droitinternational-droits-fondamentaux

Korpi, Walter. «Un État-providence contesté et fragmenté. Le développement de la citoyenneté sociale en France. Comparaisons avec la Belgique, l'Allemagne, l'Italie et la Suède». Revue française de science politique RFSP 45, n. ${ }^{\circ} 4$ (1995): 632-667. 
Koubi, Geneviève. «Vers l'égalité des chances: quelles chances en droit?». En L'égalité des chances: Analyses, évolutions, perspectives. Colección «Recherches», editado por Geneviève Koubi y Gilles Guglielmi. La Découverte, 2000.

Legendre, Myriam. «Environnement et santé». Santé Publique 15, n. 3 (2003): 291-302.

Mkandawire, Thandika. «Les politiques de développement social: un nouveau défi pour les sciences sociales». Revue internationale des sciences sociales $189, \mathrm{n} .^{\circ} 3$ (2006): 427-438.

Morin, Françoise. «Les droits de la Terre-Mère et le bien-vivre, ou les apports des peuples autochtones face à la détérioration de la planète». Revue du MAUSS 42, n. ${ }^{\circ} 2$ (2013): 321-338.

Ortiz, Jean. "Le concept andin de "buen vivir" et "l'écosocialisme"». Le Grand soir, 18 de septiembre de 2013. https:/ / www.legrandsoir.info/le-concept-andin-de-buenvivir-et-l-ecosocialisme.html

Pawin, Rémy. «Le bien-être dans les sciences sociales: naissance et développement d'un champ de recherches». L'Année sociologique 64, n. ${ }^{\circ} 2$ (2014): 273-294.

Sauvé, Jean-Marc. Avant-propos. En Conseil d'État, Étude annuelle: L'action économique des personnes publiques. EDCE, 2015.

Solón, Pablo. «Le "buen vivir", une autre vision du monde» (trad. del español por Jean Merckaert). Revue Projet 362 n. ${ }^{\circ} 1$ (2018): 66-72.

Svampa, Maristella. "Néo-"développementisme" extractiviste, gouvernements et mouvements sociaux en Amérique latine». Problèmes d'Amérique latine 81, n. ${ }^{\circ}$ (2011): 101-127.

Vanhulst, Julien y Adrian E. Beling, «Buen vivir et développement durable: rupture ou continuité?». Écologie E politique 46, n. ${ }^{\circ} 1$ (2013): 41-54. 
Geneviève Koubi

\section{Sobre el autora}

Profesora principal de la Universidad de París-Vincennes-Saint Denis (Francia), doctora en Derecho Público por la Universidad de Montpellier I (1984) y doctora en Derecho por la Universidad de París-Sorbona (1978). Asimismo, es directora del Centre d'études et de recherches Fondements du droit public (CERFDP) de la Universidad Cergy-Pontoise y directora del laboratorio Forces du Droit: Paradoxes, Comparaisons, Expérimentations. 